\title{
Method for Detection of Foreign Matters Contained in Dryed Nori (Seaweed) based on Optical Property
}

\author{
Transparent Foreign Matter Detection by using Bidirectional Reflectance \\ and Polarization Characteristcs
}

\author{
Kohei Arai \\ Science and Engineering Faculty, Department of Information Science \\ Saga University \\ Saga City, Japan
}

\begin{abstract}
Optical property, such as spectral reflectance, bidirectional reflectance distribution functions and polarization characteristics of dried seaweeds is clarified together with an attempt for transparent foreign matter detection considering optical property of dried seaweeds, such as spectral transparency, bidirectional reflectance and transparency as well as polarimetric properties. Through experiments, it is found that transparent foreign matter can be detected by using bidirectional reflectance distribution function as well as polarization characteristics.
\end{abstract}

Keywords-Seaweeds; optical characteristics; BRDF; polarization characteristics; foreign matter detection

\section{INTRODUCTION}

Nori (Porphyra Yezoensis ${ }^{1}$ ) of seaweed is the famous product for Japanese traditional food in particular. Seaweed rolls are getting popular in the world. One of the problems in Seaweed production is foreign matter detection in particular, transparent foreign matters. It is relatively easy to detect nontransparent foreign matters such as fishing hook, gravel and so on. By using the difference between transparent of the seaweed products, foreign matters can be detected. Most of the foreign matter detection machines use such difference of the transparency. If the transparency of seaweed is smaller than the previously determined threshold, such seaweed has to be removed from the seaweed product for sale. It, however, is comparatively difficult to detect transparent foreign matters such as a peace of tegus (fishing line fragment).

The optical properties of dried seaweed are not necessarily clarified, and it is not clear whether it is useful for detecting foreign matter mixed with any optical characteristics. In addition, it is difficult for transparent foreign matters such as tegus to detect contaminants contaminated into dried seaweed by optical characteristics.

Conventional methods for foreign matter detection use just reflectance of the dried nori in concerns at a certain wavelength. It, however, is difficult to detect transparent foreign matter, in particular, such as tegus. In this paper, the spectral reflectance and transparency characteristics, bidirectional reflectance and transparency characteristics and polarization characteristics of dried seaweed is clarified. Then, a detection method of the inclusion of transparent foreign matters is proposed based on these characteristics.

The following section describes fundamental optical characteristics of dried seaweeds. Then the proposed method for foreign matter detection by using such optical properties is described followed by the experimental configuration and experimental results. Ultimately, conclusion is described with some discussions.

\section{OPTICAL CHARACTERISTICS OF A TYPICAL DRIED SEAWEED}

\section{A. Test Piece of the Typical Dried Seaweed and the Measuring Instrument used as well as Fundamental Optical Characteristics of the Test Piece Measured in the Laboratory}

Spectral reflection characteristics and its angle dependency (bidirectional reflectance characteristics: BRDF (Bi-Directional Reflectance Distribution Function)) [1]-[4] as well as, s and p polarized radiance of front and back sides of representative dried seaweed is measured using spectral radiometer MS-720 (spectral sensitivity ranges from $350 \mathrm{~nm}$ to $1050 \mathrm{~nm}$ ). The major specification of MS-720 is shown in Table I while the outlook of the MS-720 is shown in Fig. 1.

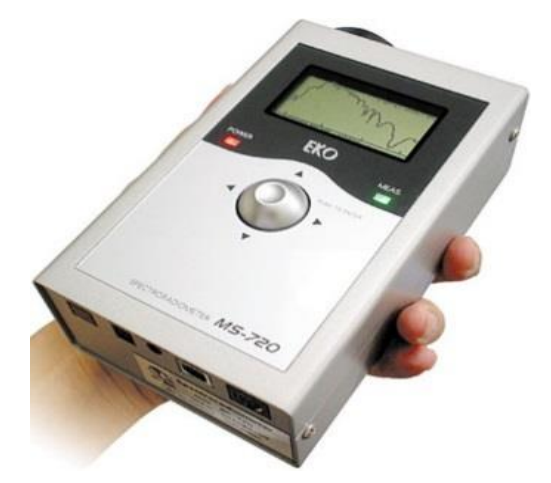

Fig. 1. Outlook of the MS-720.

\footnotetext{
${ }^{1}$ http://en.wikipedia.org/wiki/Porphyra

This research is conducted with Nishihatsu Co. Ltd. in Saga, Japan as a cooperative study between the company and Saga University.
} 
TABLE I. MAJOR SPECIFICATION OF MS-720 OF SPECTRORADIOMETER

\begin{tabular}{|l|l|}
\hline Wavelength range & $350 \sim 1,050 \mathrm{~nm}$ \\
\hline Wavelength Interval & $3.3 \mathrm{~nm}$ \\
\hline Wavelength Resolution & $10 \mathrm{~nm}$ \\
\hline Wavelength Accuracy & $<0.3 \mathrm{~nm}$ \\
\hline Aperture Angle(Full) & $180^{\circ}$ \\
\hline Straylight & $<0.15 \%$ \\
\hline Temperature Dependency & $\pm 5 \%$ \\
\hline Output Unit & $\mathrm{W} / \mathrm{m}^{2} / \mu \mathrm{m}$ \\
\hline Measuring Interval & $0.005 \sim 5 \mathrm{sec}($ Auto $)$ \\
\hline Size & $\mathrm{W} 100 \times \mathrm{D} 165 \times \mathrm{H} 60(\mathrm{~mm})$ \\
\hline Weight & $720 \mathrm{~g}$ \\
\hline Interface & $\mathrm{RS}-232 \mathrm{C} / \mathrm{USB}$ \\
\hline
\end{tabular}

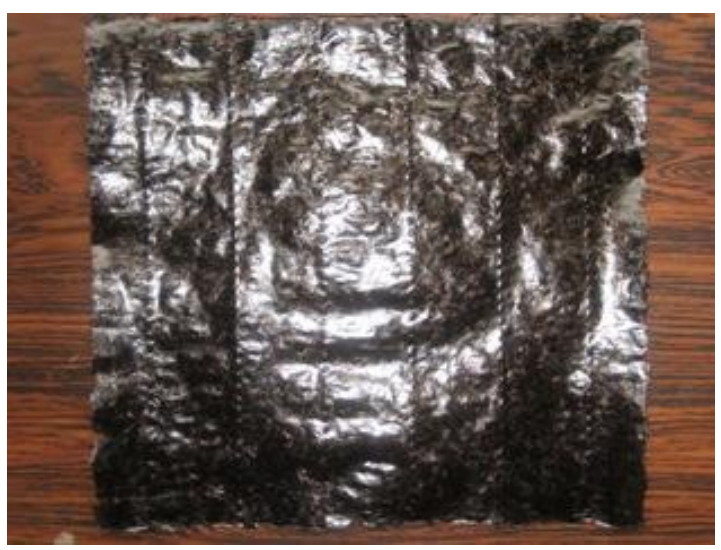

Fig. 2. The typical dried seaweed used.

The reflectance and linear polarization degree of the component are measured. A polarizer is attached to the front aperture of the optical system of the spectroradiometer so that the deflection angle can be changed.

Also, incidence angle can be changed. The typical dried seaweed used for experiments is shown in Fig. 2.

It shows the front side of the dried seaweed. Therefore, it is a little bit glossy partially. The reflectance and the degree of polarization is measured at the non-glossy portion of the dried seaweed. The degree of linear polarization DP [5]-[7] can be defined by (1).

$$
\mathrm{DP}=\left(\mathrm{R}_{\max }-\mathrm{R}_{\min }\right) /\left(\mathrm{R}_{\max }+\mathrm{R}_{\min }\right)
$$

Where, $\mathrm{R}_{\max }$ and $\mathrm{R}_{\min }$ denote maximum and minimum radiance, respectively.

\section{B. Measured Spectral Reflectance, BRDF, Degree of Polarization}

The measured spectral reflectance is shown in Fig. 3. In the figure, the observation angle is represented by the zenith angle. Also, Transparency, $\mathrm{R}_{\mathrm{t}}, \mathrm{R}_{\mathrm{h}}, \mathrm{R}_{\mathrm{s}}, \mathrm{R}_{\mathrm{p}}, \mathrm{R}_{15}, \mathrm{R}_{30}, \mathrm{R}_{45}, \mathrm{R}_{60}, \mathrm{DP}$ denotes Transparency, Reflectance of the tail (back) seaweed surface, Reflectance of the head (front) seaweed surface, Reflectance for s polarization, Reflectance for $\mathrm{p}$ polarization, Reflectance at zenith angle of 15, 30, 45, and 60 degrees, and Degree of Polarization: DP defined as (1), respectively.

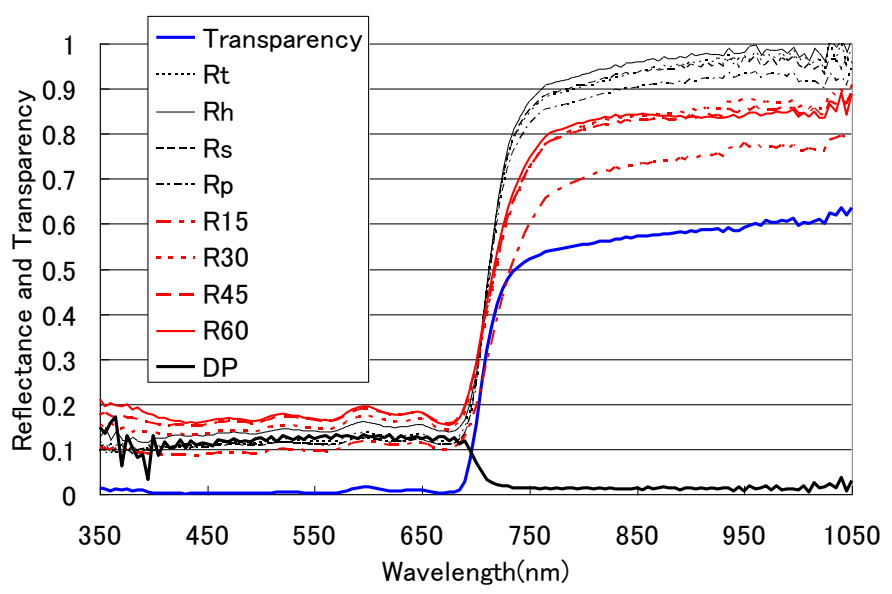

Fig. 3. Spectral reflectance as a function of observation angle and transparency as well as s and p polarized reflectance together with degree of polarization of the typical dried seaweed.

Depending on the moisture content of dried seaweed, in particular, the reflectance in the absorption region of water at $940 \mathrm{~nm}$ is largely different. It is also possible to estimate the moisture content using this characteristic.

The reflectance in the wavelength range from the ultraviolet to the red edge is as low as 0.2 or less, but extremely high in the near infrared wavelength range. The transparency is also extremely low in the wavelength range from the ultraviolet to the red edge, but it is understood that it is translucent in the near infrared wavelength range. Therefore, it is possible to utilize the difference in transparency as well as the reflectance of dried seaweed with no foreign matter to detect transparent contaminants. In addition, the difference in reflectance on the back and front side of dried seaweed shows that the reflectance of the glossy surface nori surface is somewhat higher than that of the large surface roughness back-seaweed surface.

BRDF of dried seaweed can be calculated with the measured reflectance at the different observation angles. Fig. 4 shows the calculated BRDF. BRDF may change when the target dried seaweed contains foreign matter. Therefore, there is a possibility of foreign matter detection based on BRDF measurements.

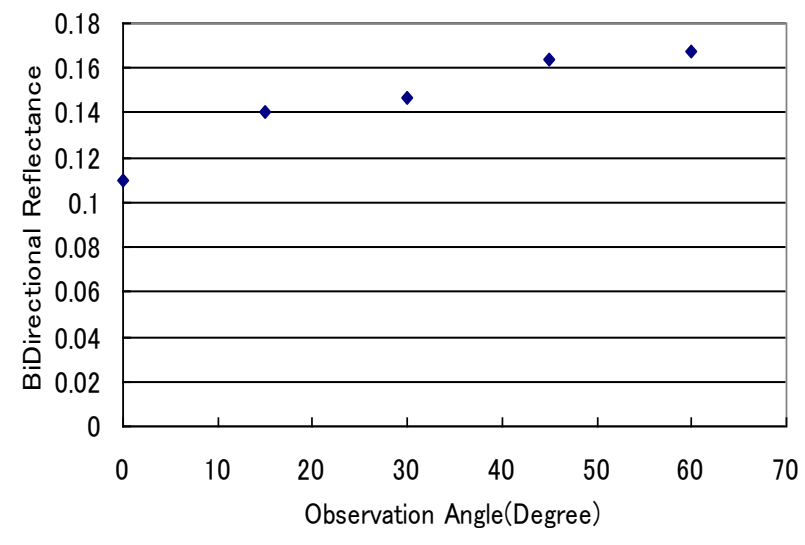

Fig. 4. BRDF of the typical dried seaweed. 


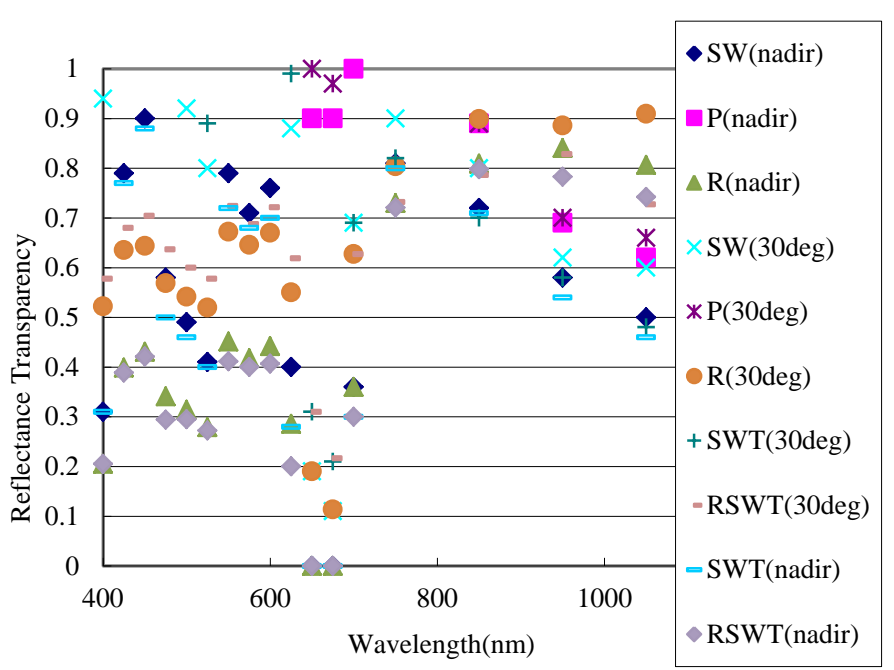

Fig. 5. Reflectance and transparency of dried seaweed at the different viewing angles, nadir and 30 degrees.

Spectral reflectance of the dried seaweed at the different viewing angles, nadir and 30 degrees is shown in Fig. 5. In this case, CCD cameras are situated in the nadir and 30 degree of nadir angle. Therefore, reflectance in the directions of nadir and 30 degree can be measured.

In the figure, SW, P, R denotes the incident and reflected radiance, respectively. Also, The number in the bracket denotes viewing angle, nadir and 30 degrees. For instance, RSWT(nadir) denotes the reflectance of the dried seaweed containing tegus in the nadir direction while RSW denote the reflectance of the dried seaweed without tegus in the nadir direction.

Also, the difference between "delta(nadir): R and RSWT" as well as "delta(30deg): SWT and R" can be evaluated together with "the effect" of the difference between the differences. Also, the difference between "delta'(30deg): RSWT(30deg) and R(30deg)" is shown in Fig. 6. Therefore, the effect of BRDF for foreign matter detection can be assessed.

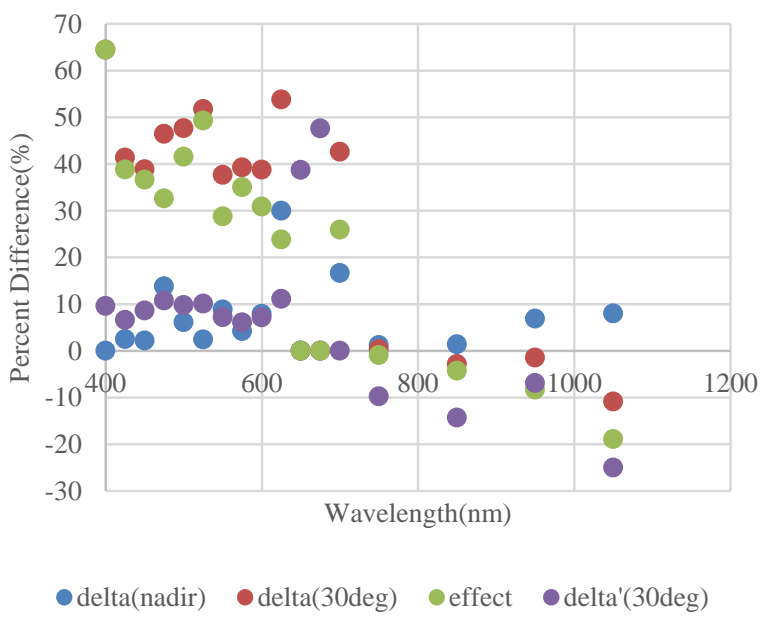

Fig. 6. Effect of BRDF for foreign matter detection.
Also, spectral reflectance of the typical dried seaweed is measured as a parameter of polarization angles ranged from 220 to 100 degrees with 20 degrees of step. Fig. 7 shows the result. Then, DP is calculated with the measured reflectance. The DP is also shown in the same figure. From Fig.7, it can be seen that dry seaweed has DP less than 0.1 from ultraviolet to red edge, but has almost no polarization characteristics in the near infrared wavelength range. Furthermore, when the observation angle is varied, it can be confirmed that the reflectance at the observation zenithal angle of 0 degree, that is, the reflectance in the direct downward direction is the lowest, and the reflectance becomes higher as the languidness increases.

When the reflectance at $500 \mathrm{~nm}$ is expressed as a function of the observation zenith angle, it is as shown in Fig.8, and it is understood that the BRDF characteristic of the dry seaweed surface is not Lambertia ${ }^{2}$, but follows the Minnerart Reflection Model $^{3}$ [1]. The reflectance varied periodically with the 180 degree of polarization angle.

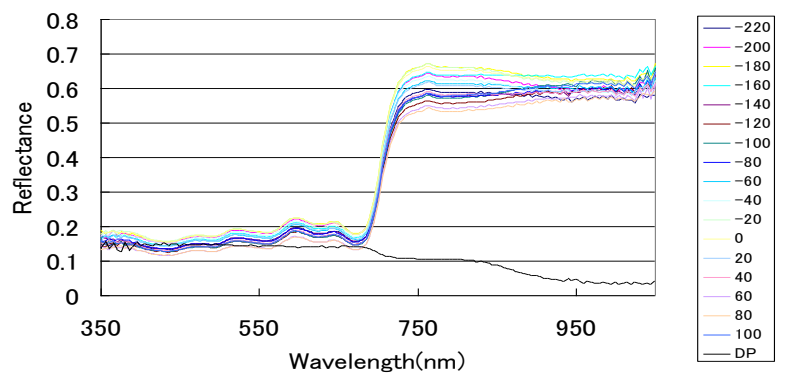

Fig. 7. Spectral reflectance as a function of polarization angle and degree of polarization of the dried seaweed.

These are fundamental optical properties of the dried seaweed. Foreign matter contained in the dried seaweed is intended to detect based on the optical properties. Namely, the BRDF of the seaweed containing foreign matter is different from the BRDF without foreign matter. Also, the DP with foreign matter differ from the DP without foreign matter. Transparency difference between seaweed with and without foreign matter can be used for foreign matter detection. This is the fundamental idea of the proposed method for foreign matter detection.

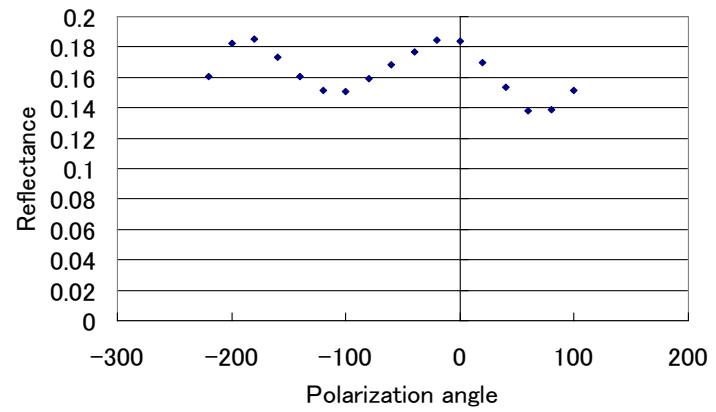

Fig. 8. Reflectance at $500 \mathrm{~nm}$ as a function of polarization angle.

\footnotetext{
${ }^{2}$ https://en.wikipedia.org/wiki/Lambert\%27s_cosine_law

${ }^{3} \mathrm{http}: / /$ thesai.org/Downloads/IJARAI/Volume2No9/Paper_4-Bi-

Directional_Reflectance_Distribution_Function.pdf
} 
From these measurement results and estimation results:

1) Reflectance and transmittance in the near-infrared wavelength range are particularly effective for detecting foreign matter mixed in dry seaweed.

2) The bidirectional reflectance characteristic is useful for detecting transparent foreign matters such as tegus.

3) Linear polarization degree is also effective for detection of transparent foreign matters.

\section{PRACTICAL EXPERIMENTS}

\section{A. Measurement Configuration}

Foreign matter containing dried seaweed is placed at 40 degrees for the light source and 70 degrees for the surface reflection observation camera with respect to the dried seaweed surface. The waveforms of the two front reflection cameras are compared. Also, the bidirectional reflectance is measured by similarly arranging the table reflection observation camera at 100 degrees. The measurement configuration is shown in Fig. 9.

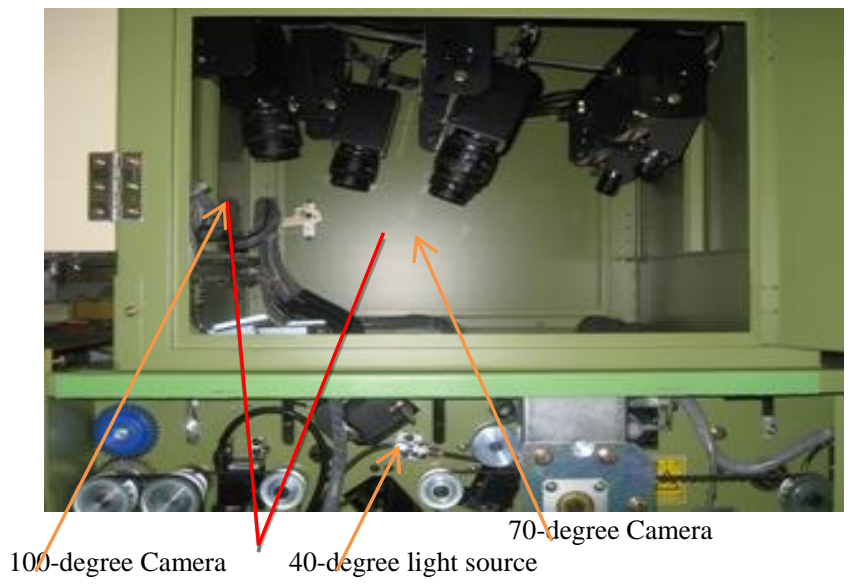

Fig. 9. Measurement configuration (Side view).

The table reflector lights the inverter with a fluorescent lamp of 20W (3 wavelength daylight color). A polarizing film is attached to the glass surface. As shown in the photograph of Fig. 10, when the polarization film is shifted by 90 degrees on the light source, light is not transmitted.

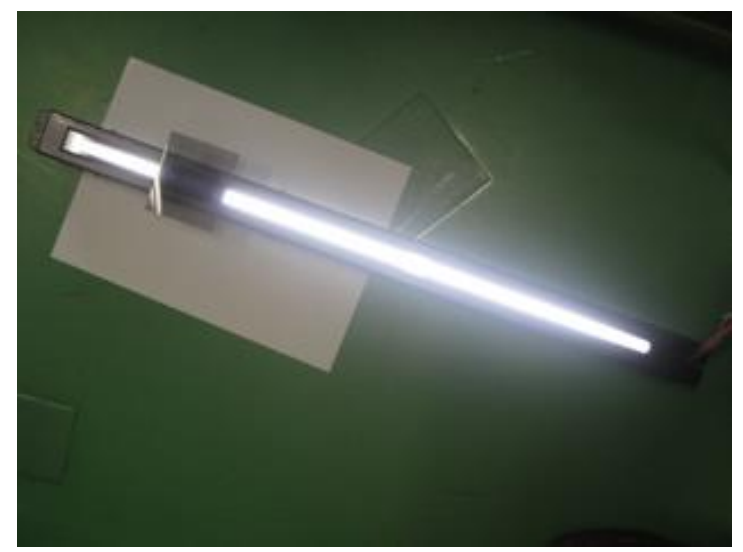

Fig. 10. Light source used (20W three wavelength combined quasi natural light source with polarization film).

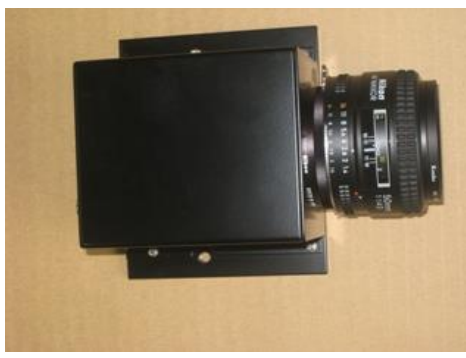

Fig. 11. CCD camera used.

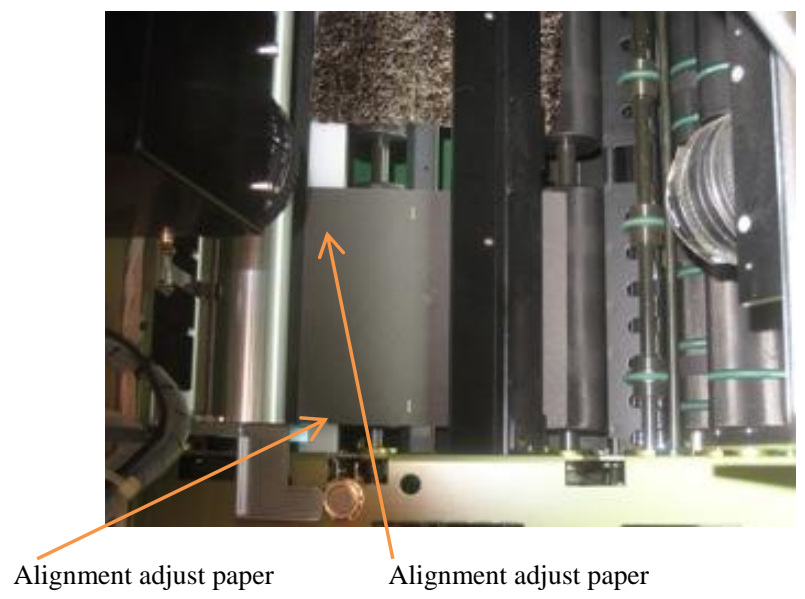

Fig. 12. Measurement configuration (Top view).

For the table reflection camera, the CCD line camera (black and white) in Fig. 11 is used. A polarizing film is attached to the CCD portion to be orthogonal to the fluorescent lamp light source.

A top view of the measurement configuration is shown in Fig. 12. In the figure, standard white paper placed at the reference position for optical axis adjustment is situated. Also, on the top of it is placed the test piece of dried seaweed containing transparent foreign matter. When detecting the contamination of actual dried seaweed contamination, the dried seaweed is moved at a high speed in this.

To match the optical axis of the 70 and 100-degree camera, as shown in Fig. 13, the optical axis adjustment paper is affixed to the reference position on the dried seaweed surface. While monitoring the camera output, the optical axis is adjusted so that the output positions when both cameras saw the same optical axis adjustment paper are matched. Fig. 14 shows the waveform of the camera output in optical axis adjustment.

We set the reference sensitivity reference color as the reference to Japan Painting Association No. C22-40D (Munsell number 2.5Y4 / 2) shown in Fig. 15, put the reference color on the optical axis of the table reflection camera, are adjusted to have the same sensitivity (waveform voltage). At that time, the sensitivity is adjusted to be 33 .

Milky white tegus with thickness of about $0.2 \mathrm{~mm}$ and length of $35 \mathrm{~mm}$ are used for testing. Fig. 16 shows this Tegus. The "U"-shaped tegus is placed on the dried seaweed in the state of $0.2 \mathrm{~mm}$ in thickness and $35 \mathrm{~mm}$ in length on the optical axis. The camera output waveform is shown in Fig. 17. 


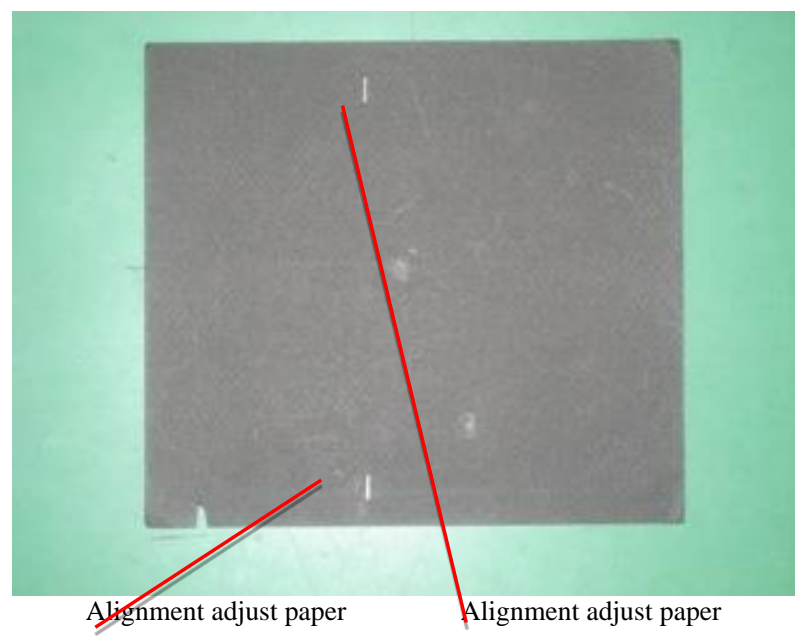

Fig. 13. Alignment adjust for both of cameras with two white alignment adjust papers.

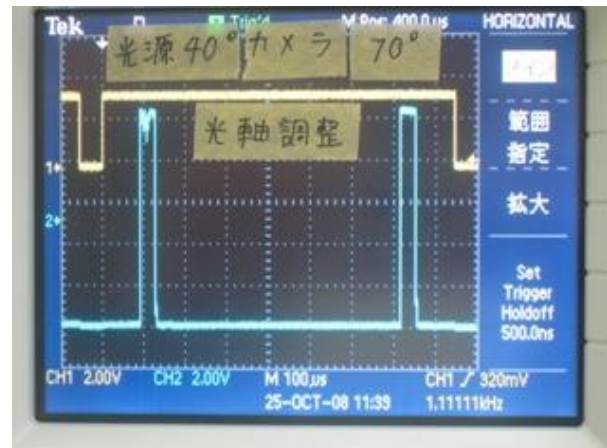

(a) 70-degree camera

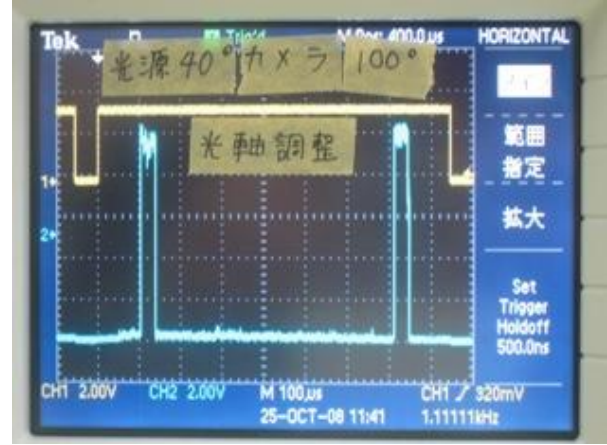

(b) 100-degree camera

Fig. 14. Camera output when alignment adjustment for both 70 and 100degree cameras.

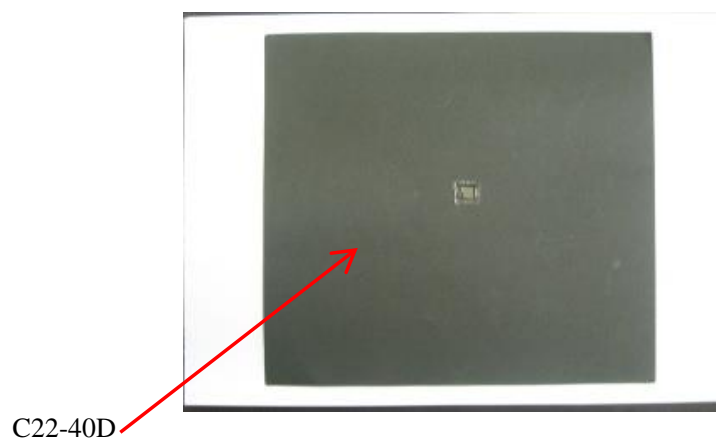

Fig. 15. Standard color of C22-40D.

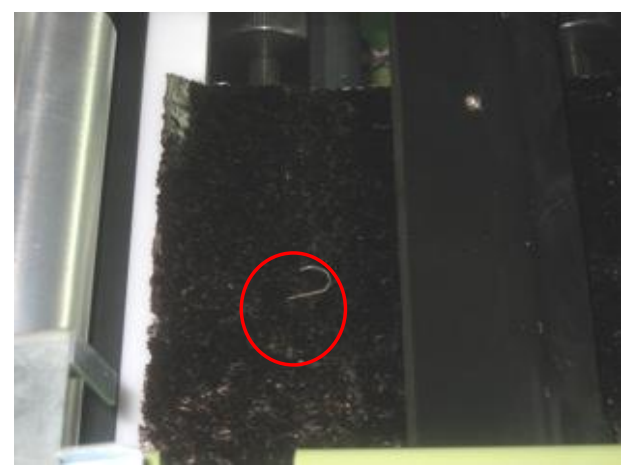

Fig. 16. Transparent fish line used (in the red circle).

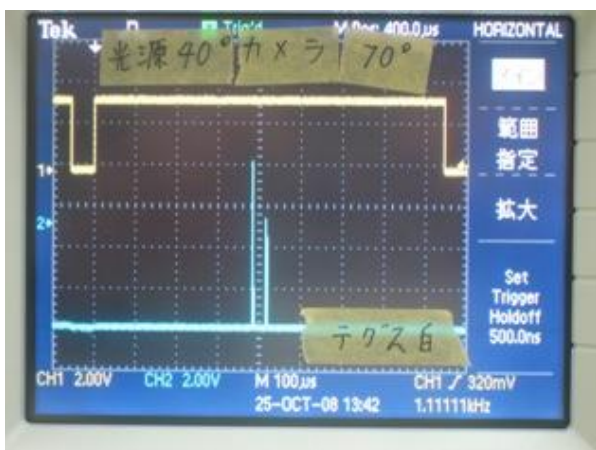

(a) 70-degree camera

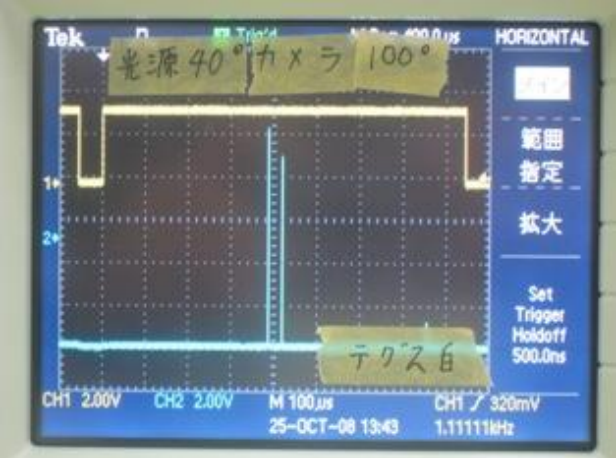

(b) 100-degree camera

Fig. 17. Camera output from the transparent fish line on the head surface of the dried seaweed.

Reflected light from the tegus differs by 1.25 times between the 70-degree camera and the 100-degree camera because the BRDF changes (sensitivity is higher because the output of the 100-degree camera is closer to specular reflection), it is confirmed that foreign matter detection is possible.

Table II also shows the outputs of the 70-degree camera and the 100-degree camera and their ratios when the position of the tegus is changed. From this table, it is found that foreign matter detection is possible regardless of the position of the tegus.

The surface roughness of the backside of dried seaweed is relatively high. Therefore, the foreign matter detection capability is confirmed when foreign matter such as Tegus is mixed in or sticking to it. Fig. 18 shows the dried seaweed that Tegus sticks to the reverse side. 
TABLE II. CAMERA OUTPUT DIFFERENCE BETWEEN 70 AND 100 DEGREE CAMERAS WHEN BOTH CAMERAS OBSERVE THE SAME FISH LINE (LOCATIONS ARE DIFFERENT)

\begin{tabular}{|l|l|l|l|l|l|l|}
\hline & \multicolumn{5}{|l|}{ Sensitivity at the various positions } & Mean \\
\hline 70 Deg. & 34 & 20 & 36 & 25 & 34 & 29.8 \\
\hline 100 Deg. & 144 & 25 & 134 & 53 & 114 & 94 \\
\hline 100 Deg./70 Deg. & 4.2 & 1.3 & 3.7 & 2.1 & 3.4 & 3.2 \\
\hline
\end{tabular}

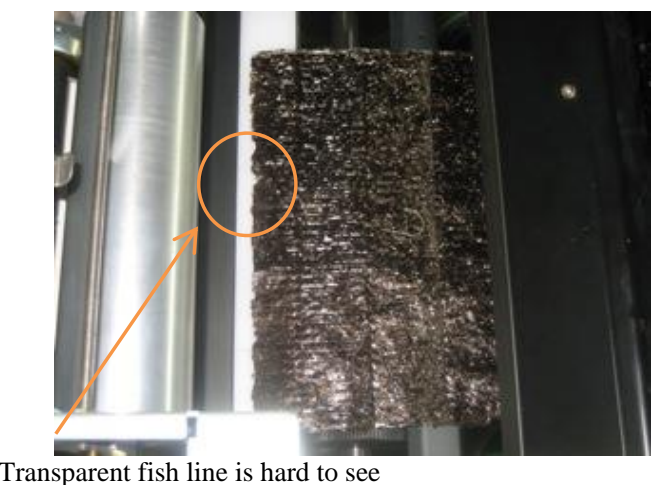

Fig. 18. Dried seaweed with transparent fish line attached to the tale surface.

Fig. 19 shows the camera output from the transparent fish line on the tale surface (backside) of the dried seaweed. It is clearly seen that the Tegus containing dried seaweed can be detected.
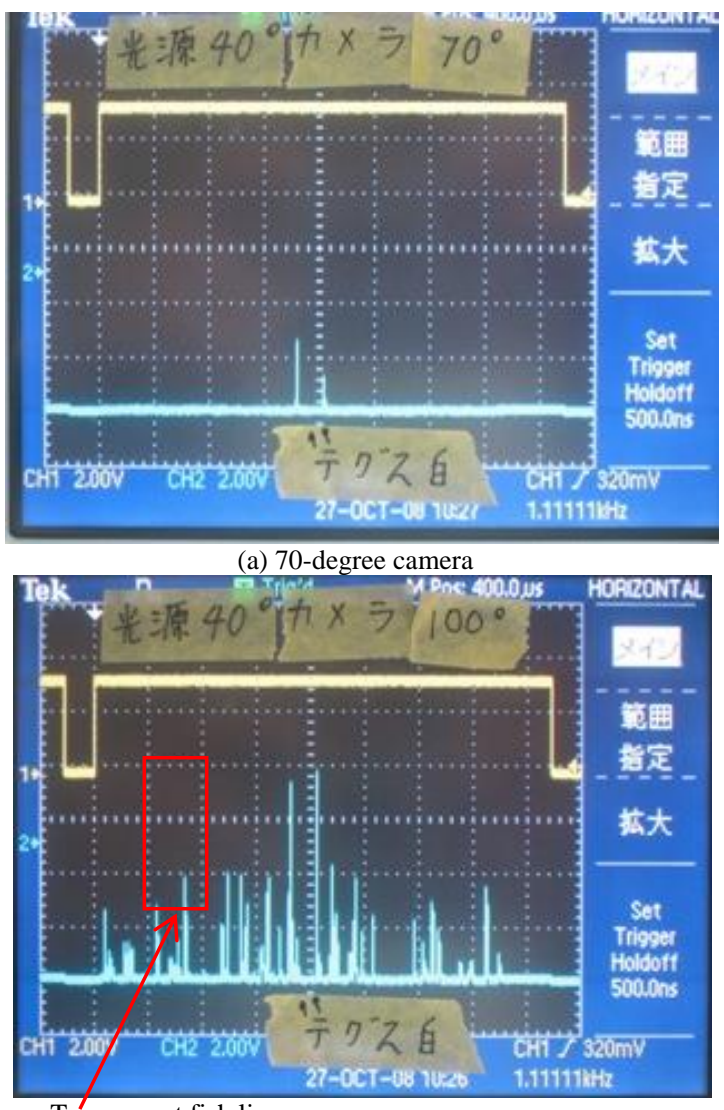

Transparent fish line

(b) 100-degree camera

Fig. 19. Camera output from the transparent fish line on the tale surface of the dried seaweed.
In the case of the camera angle of 70 degrees, the waveforms of the tegus are clear compared to other parts. There is no influence on the output waveform of backside roughness of dried seaweed. In the case of the camera angle of 100 degrees, the mountain portion of the roughness of the backside of dry seaweed appears in the corrugation. Therefore, it is difficult to discriminate between the tegus and the dried seaweed. Despite sticking the polarizing film at 90 degrees to the light source part and the camera part to suppress the reflection of the dried seaweed. Therefore, it is not satisfactory to suppress the influence of the roughness of the backside of the dried seaweed.

\section{CONCLUSION}

As a method for detecting a contaminant accompanied with undulations contained in a sheet-like foreign matter of dried seaweed, a method of judging by using reflectance in two directions (bidirectional reflectance) is proposed. Light is irradiated from the light source to the sheet-like foreign matter of dried seaweed, and the reflected light is received from at least two directions. The moisture content is investigated by using a visible near infrared and short wavelength infrared CCD array for this light receiving element. As a result, foreign matters with undulations contained in the liquid foreign matter of seaweed can also be detected, and the moisture content is also measured.

Provided is a foreign matter detection device capable of accurately detecting a foreign matter which is difficult to detect even when it is mixed in a measurement object of dried seaweed. The reflectance of the object to be measured is obtained by using the oblique direction light receiving element of CCD camera, whereas in the case of the direct light receiving element of $\mathrm{CCD}$ camera, the reflectance with substantially no difference is obtained, whereas in the case where a foreign matter which is difficult to detect is mixed. In addition, the reflectance is obtained using the upward direction light receiving element of $\mathrm{CCD}$ camera with respect to the portion where the reflectance is obtained by the oblique direction light receiving element of CCD camera, and the reflectance is obtained (oblique direction reflectance/right upward direction (Reflectivity in oblique direction/reflectance in the upward direction) compared with the case in which a foreign matter is not mixed (determination of reflectance) to judge the presence or absence of contamination of foreign matters, and it is simply compared with the case of comparing the reflectance of the measurement object. Therefore, it is confirmed that foreign matters can be accurately detected.

According to the experimental results using the difference in reflectivity between directly nadir and 30 degrees, it is found that the difference in the presence/absence of tegus due to the conventional method (direct reflectance) is insignificant. Enlarged, detection capability of Tegus is improved with the proposed method by about one digit.

Experimental results, however, show that the surface reflection camera installed at 100 degrees can detect tegus when the condition of dried seaweed surface is good, but it is difficult to detect the tegus when affected by dry roughness backside roughness. Further investigation is required for overcoming this matter. 


\section{ACKNOWLEDGMENT}

The author would like to thank Dr. Osamu Yamaguchi and Dr. Masanori Tsurumaru of Nishihatsu Co. Ltd. for their effort to conduct the experiments.

\section{REFERENCES}

[1] Kohei Arai, Method for estimation of grow index of tealeaves based on Bi-Directional reflectance function: BRDF measurements with ground based network cameras, International Journal of Applied Science, 2, 2, 52-62, 2011.

[2] Kohei Arai, Comparison between linear and nonlinear models of mixed pixels in remote sensing satellite images based on Cierniewski surface BRDF model by means of Monte Carlo ray tracing simulation, International Journal of Advanced Research in Artificial Intelligence, 2, 4, 1-7, 2013.

[3] Kohei Arai, Bi-directional reflectance distribution function: BRDF effect on un-mixing, category decomposition of the mixed pixel (MIXEL) of remote sensing satellite imagery data, International Journal of Advanced Research in Artificial Intelligence, 2, 9, 19-23, 2013.

[4] Kohei Arai and Long Lili, BRDF model for new tealeaves on old tealeaves and new tealeaves monitoring through B RDF measurement with web cameras, Abstract of the 50th COSPAR(Committee on Space Research/ICSU) Congress, A3.1-0008-08 ,992, Montreal, Canada, July, 2008

[5] Kohei Arai, Osamu Yamaguchi, Masanori Tsurumaru, Optical property of dryed sea weeds, Nori and detection of transparent suspected objects containing in Nori, Research Report of Science and Engineering Faculty, Saga Unievrsity Japan, 37, 2, 1-6, 2008
[6] Kohei Arai, Hajime Koshiishi, Fukazawa, Saino, Hajime Fukushima, Ishimaru, Okuda, Hiroshi Kawamura, Total System for Ocean Observation Using Remote Sensing Satellites, Journal of Japanese Society of Ocean, 1, 51-62, 1989.

[7] K.Arai, Monte Carlo ray tracing based sensitivity analysis of the atmospheric and oceanic parameters on the top of the atmosphere radiance, International Journal of Advanced Computer Science and Applications, 3, 12, 7-13, 2012.

\section{AUTHORS PROFILE}

Kohei Arai. He received BS, MS and PhD degrees in 1972, 1974 and 1982, respectively. He was with The Institute for Industrial Science and Technology of the University of Tokyo from April 1974 to December 1978 and also was with National Space Development Agency of Japan from January, 1979 to March, 1990. During from 1985 to 1987 , he was with Canada Centre for Remote Sensing as a Post-Doctoral Fellow of National Science and Engineering Research Council of Canada. He moved to Saga University as a Professor in Department of Information Science on April 1990. He was a counselor for the Aeronautics and Space related to the Technology Committee of the Ministry of Science and Technology during from 1998 to 2000 . He was a councilor of Saga University for 2002 and 2003. He also was an executive councilor for the Remote Sensing Society of Japan for 2003 to 2005 . He is an Adjunct Professor of University of Arizona, USA since 1998. He also is Vice Chairman of the Commission-A of ICSU/COSPAR since 2008. He received Science and Engineering Award of the year 2014 from the minister of the ministry of Science Education of Japan and also received the Best Paper Award of the year 2012 of IJACSA from Science and Information Organization: SAI. In 2016, he also received Vikram Sarabhai Medal of ICSU/COSPAR and also received 37 awards. He wrote 37 books and published 570 journal papers as well as 370 conference papers. He is Editor-in-Chief of International Journal of Advanced Computer Science and Applications as well as International Journal of Intelligent Systems and Applications. http://teagis.ip.is.saga-u.ac.jp/ 